\title{
Waveguide Joint Design and Validation for use in Acoustic Vector-corrected Network Analysers
}

\author{
$1^{\text {st }}$ Marcus MacDonell, Student Member IEEE $\quad 2^{\text {nd }}$ Keshav Basnet $\quad 3^{\text {rd }}$ Jonathan Scott, Senior Member IEEE \\ Department of Engineering \\ University of Waikato \\ Hamilton, NZ \\ Department of Engineering \\ University of Waikato \\ Hamilton, NZ
Department of Engineering
University of Waikato \\ Hamilton, NZ
}

\begin{abstract}
The Vector-corrected Network Analyser (VNA) has been an indispensable tool for many decades in the field of RF and microwave engineering where the availability of calibrated and traceable results is taken for granted. However, calibrated and traceable measurements are not so easily available in the acoustic domain. In an effort to allow such measurements, a dual-port acoustic vector network analyser (AVNA) has been designed and built. The calibration of this test and measurement system hinges on the repeatability of the uncalibrated system and its acoustic waveguide joints. The first generation of acoustic waveguide joints have been 3D printed with a high precision printer and designed to include alignment pins, $O$-rings, and a reinforcing 'clip' system to allow for a consistent bolt torque without damaging the plastic waveguide. We show that the resulting variation in measurements between cycles of disassembly and reassembly is acceptably small and will allow for calibration of the system. Three-dimensional printed titanium joints show increased rigidity and ease of use but similar repeatability.

Index Terms-Vector Network Analysis, Waveguide, Acoustic.
\end{abstract}

\section{INTRODUCTION}

A Vector Network Analyzer (VNA) is an instrument that measures the scattering parameters, or S-parameters, of an electrical network. S-parameters are the most commonly measured parameters because the reflection and transmission of waves from and through electrical networks are easy to measure at high frequencies. The VNA is indispensable in the fields of RF and microwave engineering. Its calibrated and traceable data is taken for granted. The authors are investigating the production of an acoustic equivalent of the microwave VNA. The project intends to build upon the decades-long investment in the technology in the microwave arena [1] to shorten the development cycle of the acoustic version. In the field of audio calibrated and traceable measurements are not so readily available. In an effort to allow such measurements a full 2-port Acoustic VNA (AVNA) has been designed and built. The calibration now hinges on the repeatability of the uncalibrated system and its waveguide joints.

Acoustic measurements to characterize materials are currently done with one of two industry standard techniques [2], [3]. Both of these methods are crudely calibrated, have not been implemented with a traceable standard, and require the

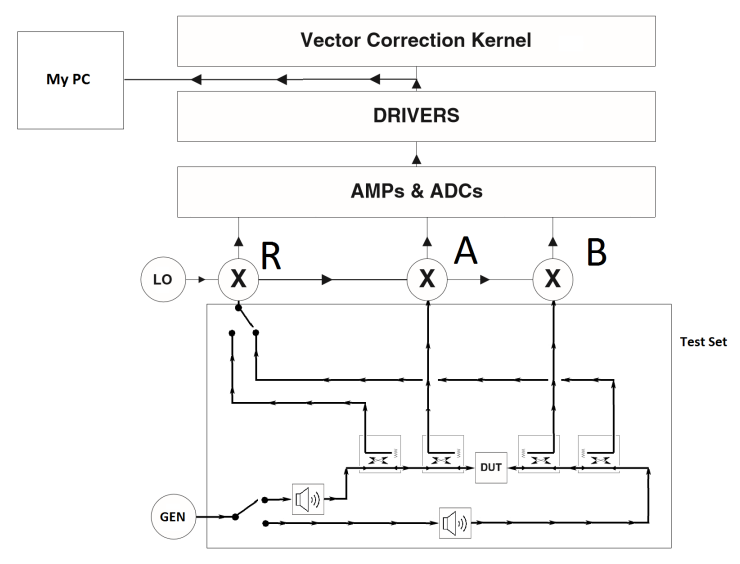

Fig. 1. A block diagram of the AVNA including signal paths, the test set, HP4395A analyser, and the PC, ultimately used for correction of the data.

operator to carry out measurement manually, frequency by frequency, in a laborious manner. Various alternative schemes have been reported in the literature to measure acoustic reflection coefficient, see for example [4], and a summary of the literature is available in [5]. In an effort to improve on these methods an Acoustic Vector Impedance Meter has been created. [5]. It was shown to measure uncalibrated acoustic $S_{11}$, the acoustic reflection coefficient. To further improve this system we are working towards using two ports, and calibrating the measurement system to permit traceable standards [6].

The accuracy of AVNA will be determined by the calibration and in turn on the system repeatibility and reliability. Once the repeatibility and reliability are establised the resulting accuracy of the calibrated AVNA is dependant on the calibration standards themselves [7]. The repeatibility of flanges is the subject of many papers in microwave engineering and many of the designs and developments to improve repeatibility have been adapted for our work in acoustics [8] [9] [10]. 


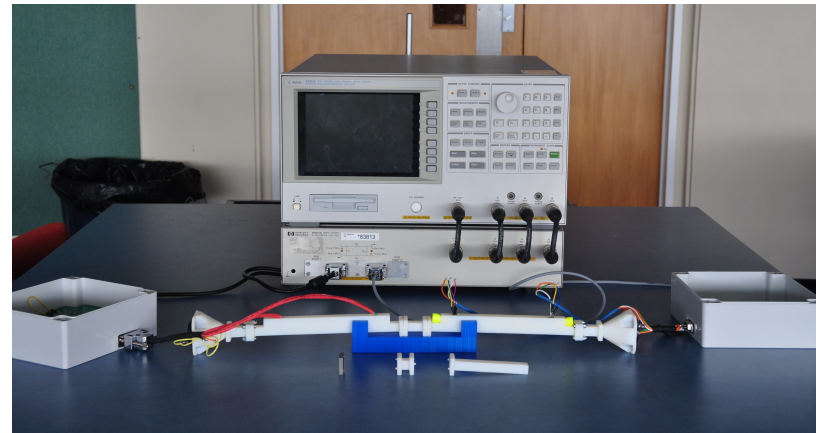

Fig. 2. The AVNA photographed with the remote heads for the $10 \mathrm{kHz}-20 \mathrm{kHz}$ range. The waveguide joints are either pressed together, or bolted without washers. (Photograph taken before the necessity for washers was understood)

\section{AVNA HARDWARE}

Figure 1 shows the block diagram of the analyser. The test set including directional couplers has been custom built with acoustic components, while the remainder of the system is provided by an Agilent 4395A Network/Spectrum Analyzer interfaced to a generic Windows-based PC. Figure 2 presents a picture of the system with remote heads having a frequency range of $10-20 \mathrm{kHz}$. The waveguide components in this case were fabricated by a resin-based Objet 3D printer.

Before addressing the development of calibration standards and algorithms it is prudent to ensure that waveguide joints are repeatable and reliable. This manuscript reports on the joint design and performance.

\section{JoINT DESIGN}

The design of the directional couplers permits a working bandwidth of a little more than one octave. For example, in in [5], [11] a range of $750 \mathrm{~Hz}-2.2 \mathrm{kHz}$, or just over 1.5 octaves, was demonstrated for the coupler design taken from [12]. Anticipating that an instrument covering the whole acoustic bandwidth will require several test sets, we have fabricated two examples covering nominal frequency ranges of $1-2 \mathrm{kHz}$ and $10-20 \mathrm{kHz}$. The lower-frequency couplers have been made in a conventional workshop using transparent acrylic. The waveguide is square with a dimension of $60.0 \mathrm{~mm}$. The higherfrequency set was initially fabricated using an Objet30 resin printer. [13] The waveguide is square with a dimension of $6.00 \mathrm{~mm}$.

Figure 3 shows the ends of two resin-printed couplers whose flanges have failed. The picture serves to show the alignment pins and the square flanges with 4 holes for bolts to secure the joint. When the bolts were torqued up, the plastic initially bulged. Investigations to select an appropriate torque setting showed that the flanges were unable to cope with any reasonable torque, and only small amounts of over-torque could do permanent damage. In order to address this problem a U-shaped washer was designed to distribute the force across the flange.

Figure 4 shows the cross section through a joint between two flanges (waveguide itself is not shown). Figure 5 makes it

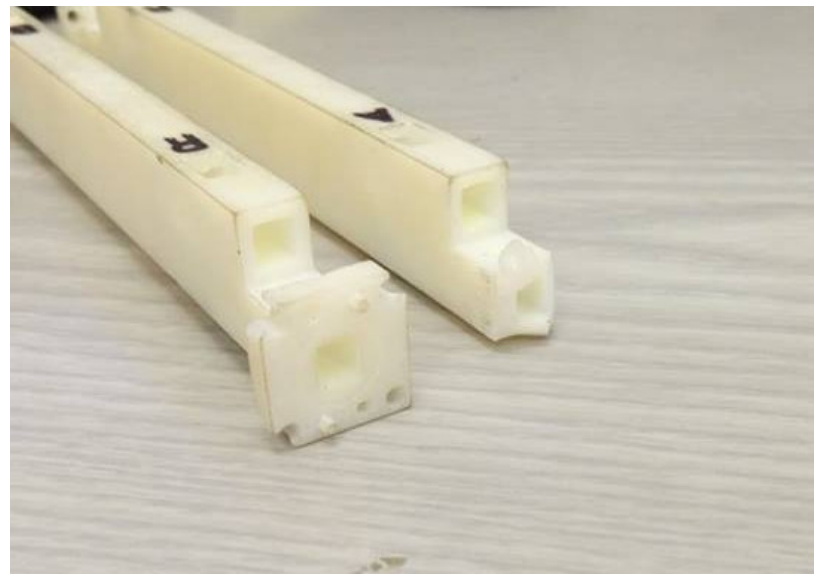

Fig. 3. Examples of broken flanges from repeated use without U-shaped washers.

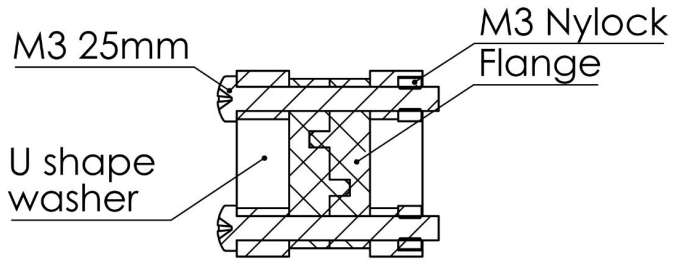

Fig. 4. Cross section diagram of two mated flanges held by bolts passing through mild steel washers. Nylon locking nuts are used with stainless steel M3 bolts.

easier to visualise by showing the outside lateral view of two couplers joined directly (a "zero-length thru"). Figure 6 shows photographs of the zero-length thru connection between two couplers fabricated in a translucent resin plastic. Also visible in the photograph are orange ear plugs used as loads in the off-axis arms of the couplers. Support legs have been added behind the washers to support the guide above the table.

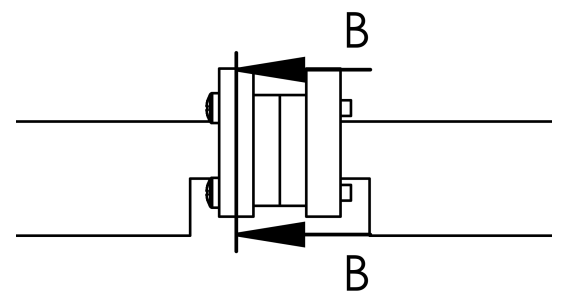

Fig. 5. Lateral view diagram where two directional couplers are joined flange-to-flange using $\mathrm{U}$-shaped washers and threaded bolts and nylon locking (nylock) nuts. The line B-B marks a cross section through one of the Uwashers. See figure 7 for the directional couplers without washers.

The joints are designed to a high precision, and include alignment pins and a slot for O-rings. In response to the difficulty of plastic flanges, we printed some components in titanium (Ti). The titanium set does not require the reinforcing washers but is a significantly more expensive waveguide material. Figure 7 shows a pair of couplers in Ti. One is presented 


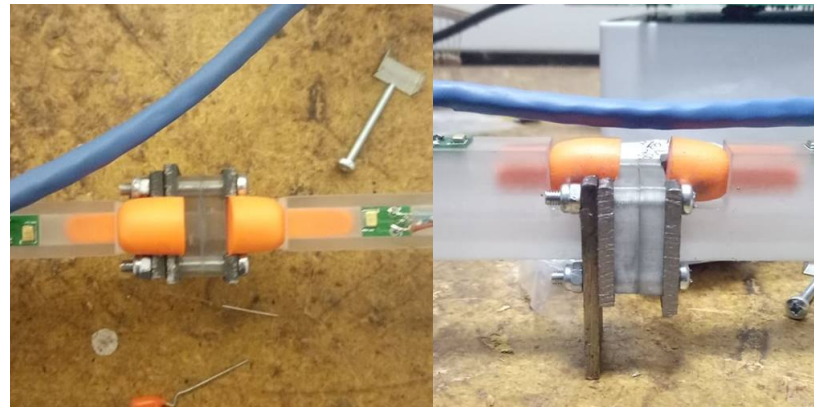

Fig. 6. Actual flange assembly. Left: Top down view, Right: Profile view. NB: mild steel U shape clips.

with an O-ring, the other without, showing the groove. In all of the small joints, a bolt torque of $0.5 \mathrm{Nm}$ is specified.

In the case of the low-frequency acrylic test set, joints are bolted together by M6 bolts and nylon locking nuts at a torque of $1.5 \mathrm{Nm}$. Generally, the flanges are sufficiently strong at this scale that full U-shaped washers are not necessary. We normally fit simple metallic washers appropriate for M6 bolts. Figure 8 shows an example waveguide component in the lowfrequency acrylic system.

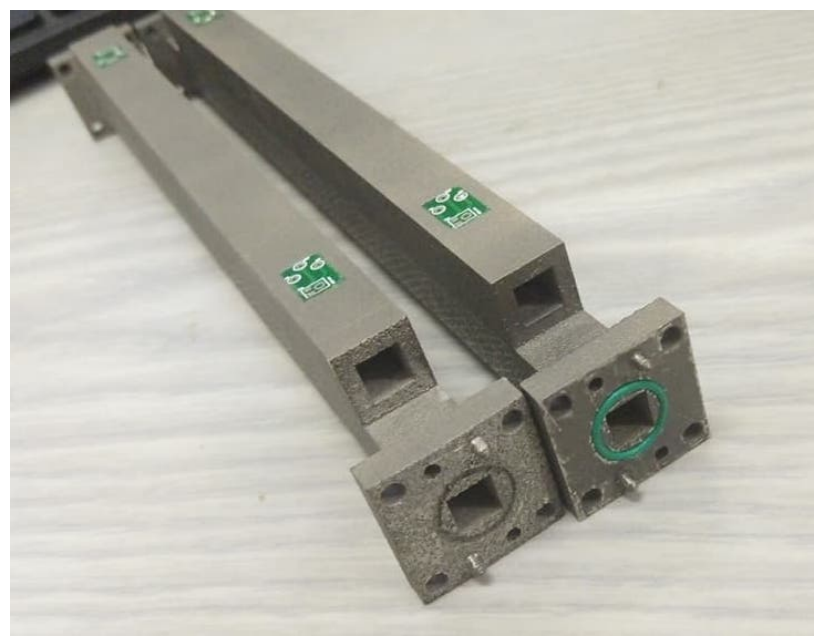

Fig. 7. The Titanium coupler set with an as sintered surface finish. The right hand coupler has an O-ring in its O-ring groove.

\section{VALIDATION}

A sample of ten measurements at each of 50 frequencies were taken in the high-frequency system without an O-ring, and another 10 sets with an O-ring. The presence of an O-ring significantly altered the measurements, so all measurements reported in this manuscript involve joints with O-rings fitted. We infer from this that the O-rings are working as intended. O-rings are specified for use in all joints.

Figure 9 exemplifies the kind of repeatability that was obtained with the printed resin guide. The figure suggests that a final accuracy of about $0.2 \mathrm{~dB}$ or just over $2 \%$ is to be expected.

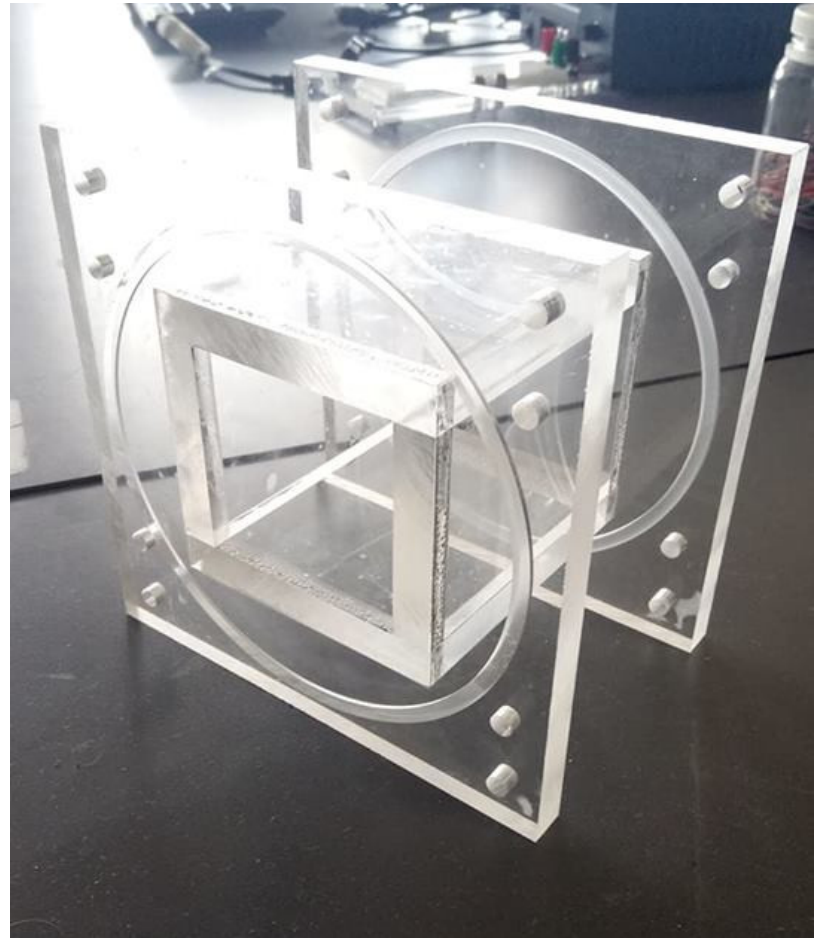

Fig. 8. Photograph of a short thru in the low-frequency, acrylic waveguide.

Surprisingly, we observe that the order in which the bolts are torqued up makes a significant difference to the repeatability. Figure 10 shows the variation in measured raw $S_{21}$ with a variety of assembly sequences. It is not worth plotting standard deviation - the gross discrepancies are visible in the raw data. It is clear that the assembly procedure matters a great deal; we suggest the bolt order depicted in figure 11 .

Figure 12 shows the same type of data for a titanium (Ti) joint as figure 9 shows for the printed resin version. It is clear that the deviation is slightly worse than the softer, resin-based joints. Figure 13 shows the Standard deviation in the large couplers for Raw S21 for three different bolt orders. Bolt orders were clockwise, or 3-2-4-1 as in Figure 10, recommended, and thirdly a $\mathrm{Z}$ or $\mathrm{N}$ shape, or 2-3-4-1 as in Figure 10. All other bolt orders are rotations or mirrors of these.

\section{CONClusion}

The repeatability of the joints in the AVNA is an a limiting factor in the overall performance of the system, including the chosen calibration algorithm. A modest torque of $0.5 \mathrm{Nm}$ is enough to cause permanent damage or total failure without washers to evenly distribute pressure over the flange, the washers also create a hard surface for the bolt/nut to press against without localized deformation of the flange. The presented plastic joint design performs relatively well, although they are sensitive to bolt order which could be due to the softness and flex of the flange material. Laser sintered Titanium versions have so far proven robust but no more repeatable than the 


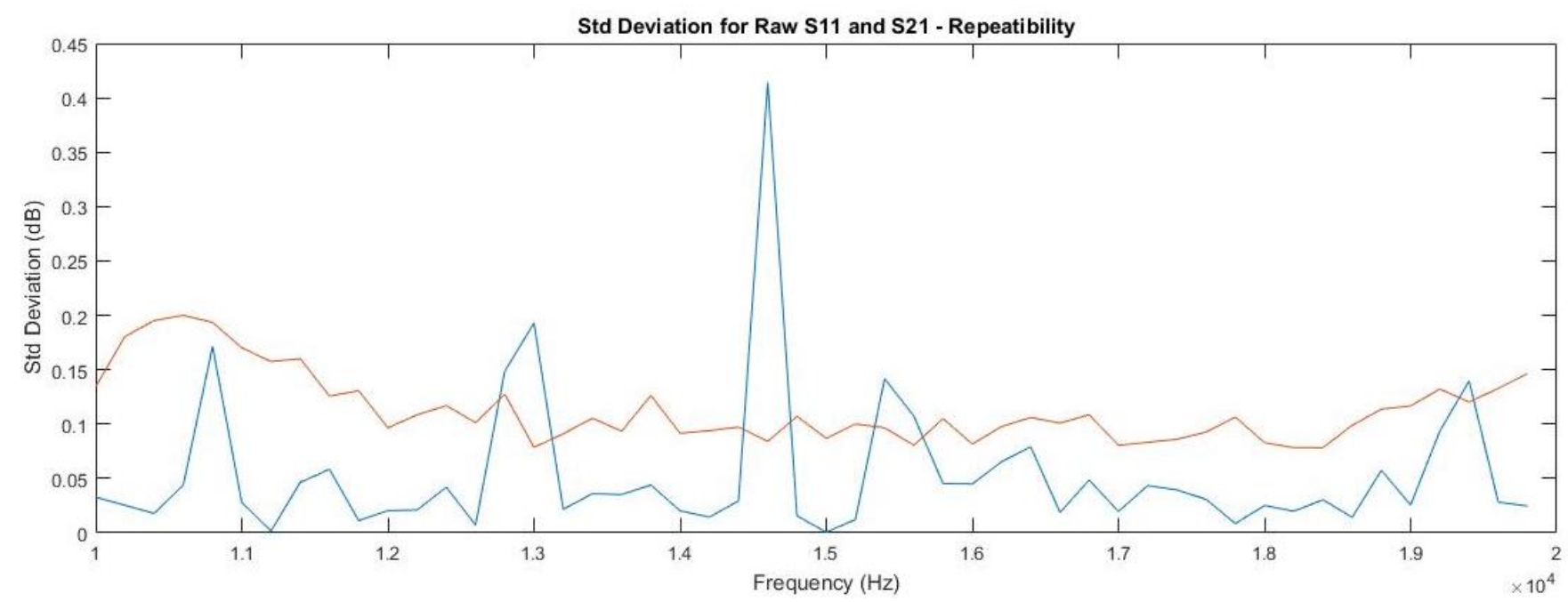

Fig. 9. Standard Deviation of raw $S_{11}$ and $S_{21}$ obtained at each measurement frequency when the joint was disassembled and reassembled ten times. The flanges were resin, the joints fitted with O-rings and U-shaped washers to distribute the torque. Bolt torque was $0.5 \mathrm{Nm}$.

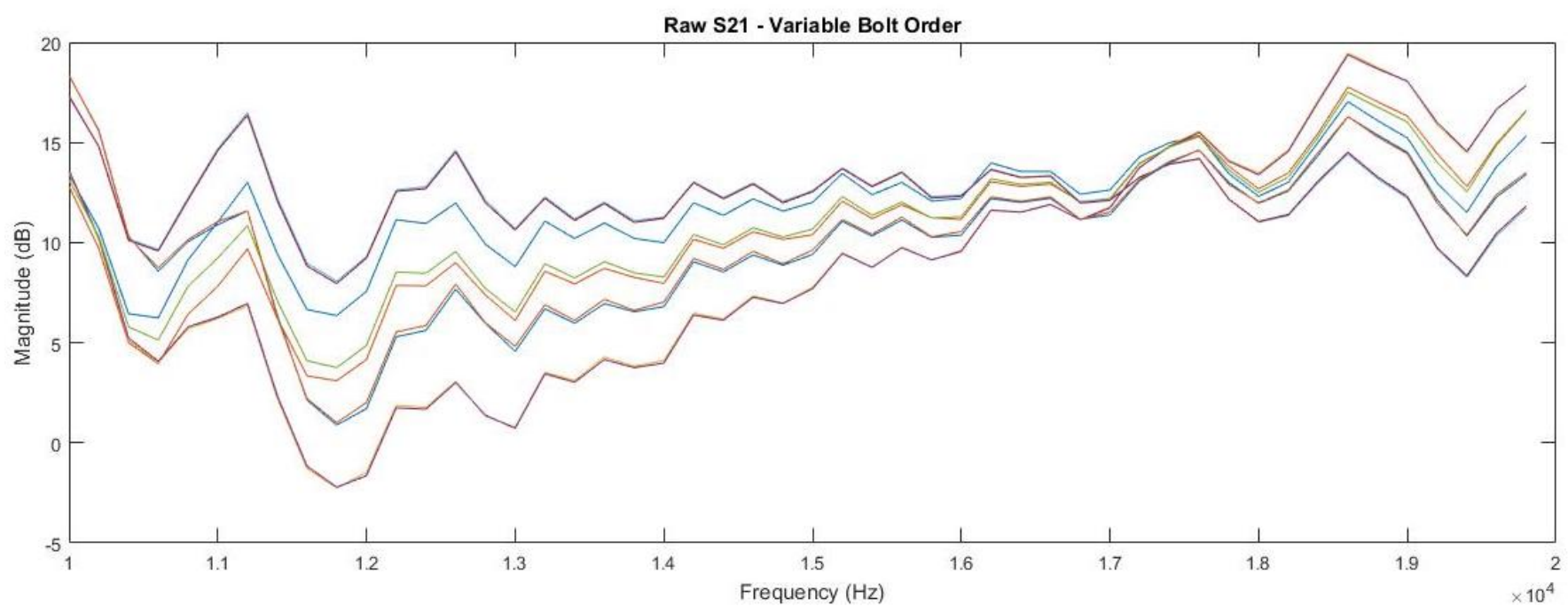

Fig. 10. Magnitude of raw $S_{21}$ measured on multiple instances of disassembly and reassembly with variable bolt order in the resin flanges.

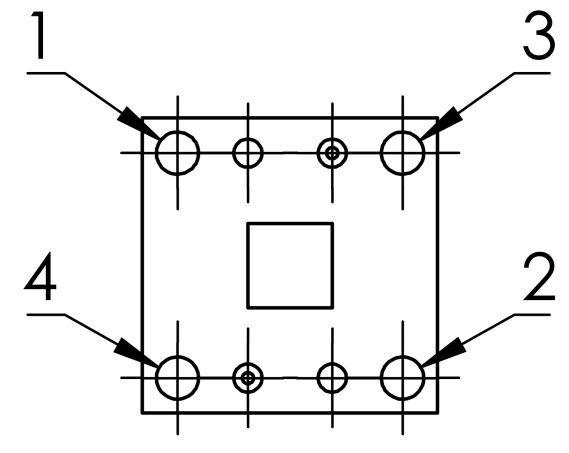

Fig. 11. Suggested bolt order for assembly of flange joints. sintered plastic type. They do eliminate the need for washers by increasing the hardness and rigidity of the flange.

\section{ACKNOWLEDGMENT}

The authors would like to thank the MBIE for funding this research as part of the Smart Ideas Fund. Thanks to Peter Higgins for workshop assistance.

\section{REFERENCES}

[1] Doug Rytting, "ARFTG 50 year network analyzer history", $71^{\text {st }}$ ARFTG Microwave Measurement Conference, 20 June 2008, Atlanta, GA, USA.

[2] ISO 10534-1:1996 Acoustics - Determination of sound absorption coefficient and impedance in impedance tubes - Part 1: Method using standing wave ratio

[3] ISO 10534-2:1998 - Acoustics - Determination of sound absorption coefficient and impedance in impedance tubes - Part 2: Transfer-function method 


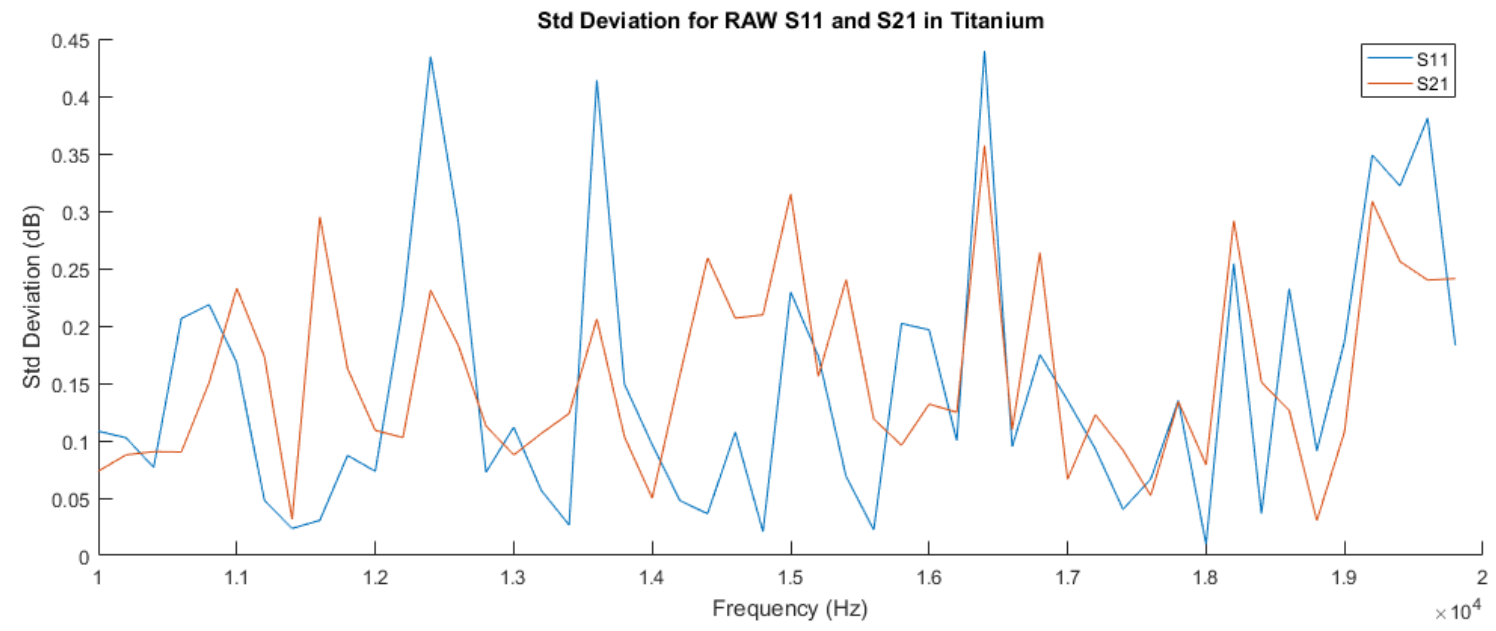

Fig. 12. Standard Deviation of raw $S_{11}$ and $S_{21}$ obtained at each measurement frequency when the joint was disassembled and reassembled ten times. The flanges were $\mathrm{Ti}$, the joints fitted with O-rings. Bolt torque was $0.5 \mathrm{Nm}$, and the bolt order was random.

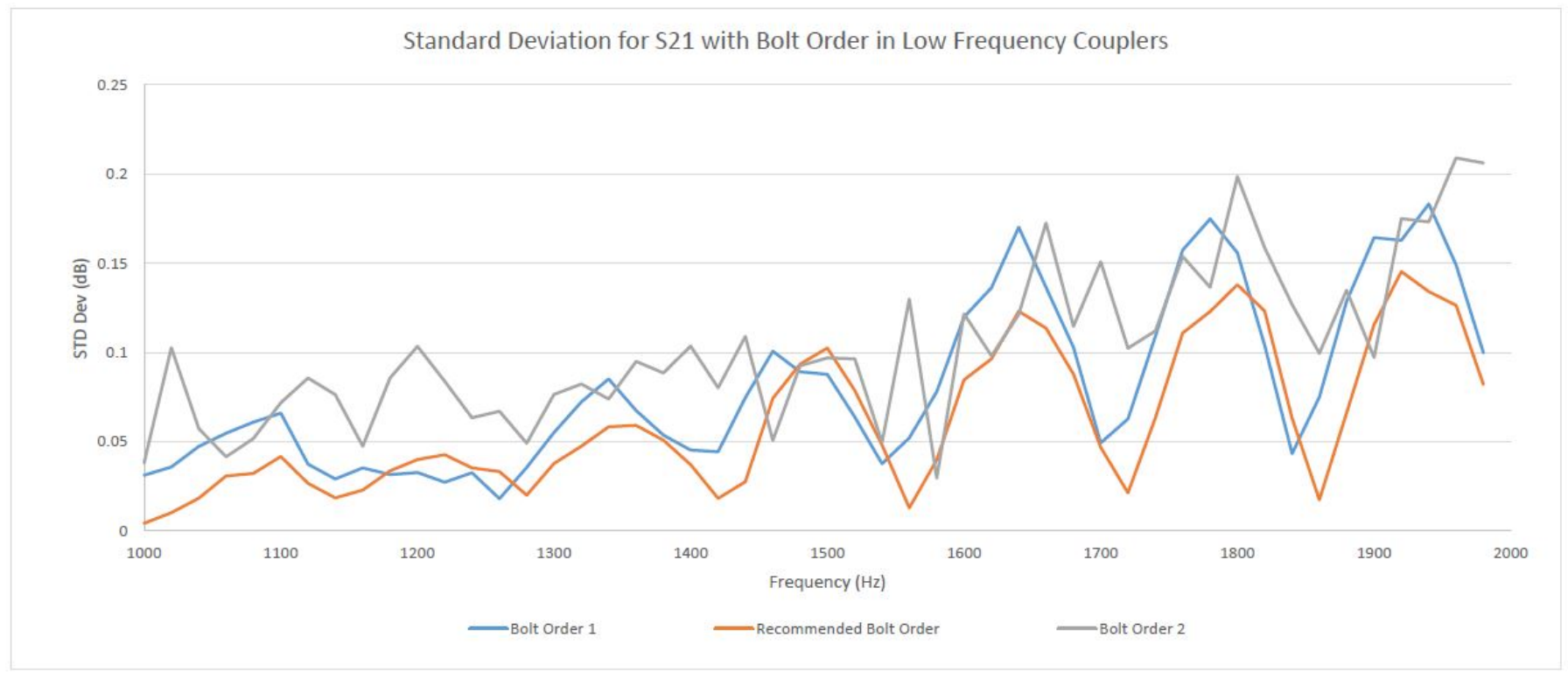

Fig. 13. Standard Deviation of raw $S_{21}$ obtained at each measurement frequency when the joint was disassembled and reassembled thirty times, with three different bolt orders (ten measurements for each bolt order).

[4] C. M. de Blok and R. F. M. van den Brink, "Direct-reading one-port acoustic network analyzer," J. Audio Eng. Soc., vol. 41, no. 4, pp. 231238, 1993.

[5] Pennington, K. (2017). Acoustic Vector Network Analyser (Thesis, Doctor of Philosophy $(\mathrm{PhD})$ ). The University of Waikato, Hamilton, New Zealand. Retrieved from https://hdl.handle.net/10289/11530

[6] Marcus, M., \& Scott, J. B. (2017). Development and Basic Calibration of an Acoustic Vector Network Analyser. Presented at the Electronics New Zealand Conference 2017 (ENZCon 2017), christchurch, New Zealand, 4-6 December 2017.

[7] D. Rytting, "Network Analyzer Accuracy Overview", 58th ARFTG Conference Digest, 2001. Available: 10.1109/arftg.2001.327486 [Accessed 16 January 2019].

[8] H. Li, A. Kerr, J. Hesler and R. Weikle, "Repeatability of waveguide flanges with worst-case tolerances in the 500\&\#x2013;750 GHz band", 79th ARFTG Microwave Measurement Conference, 2012. Available: 10.1109/arftg79.2012.6291185.

[9] M. Horibe and R. Kishikawa, "Performance of new design of waveguide flange for measurements at frequencies from $800 \mathrm{GHz}$ to $1.05 \mathrm{THz}$, 79th ARFTG Microwave Measurement Conference, 2012. Available: 10.1109/arftg79.2012.6291186.

[10] S. Rahiminejad, E. Pucci, V. Vassilev, S. Haasl, P. Kildal and P. Enoksson, "AMC pin waveguide flange for screw redundant millimeter and submillimeter measurements", 2016 87th ARFTG Microwave Measurement Conference (ARFTG), 2016. Available: 10.1109/arftg.2016.7501946

[11] Scott, J. and K. E. Pennington, "Acoustic Vector-Corrected Impedance Meter", IEEE Transactions on Instrumentation and Measurement, 2014. DOI: 10.1109/TIM.2014.2327474

[12] Lagasse, P., "Realisation of an acoustical directional coupler", Journal of sound and vibration, 15(3), April 1971, pp367-372.

[13] M. MacDonell, 'Scaling acoustic directional couplers using 3D printing', Thesis, Master of Engineering (ME), University of Waikato, 2015. 